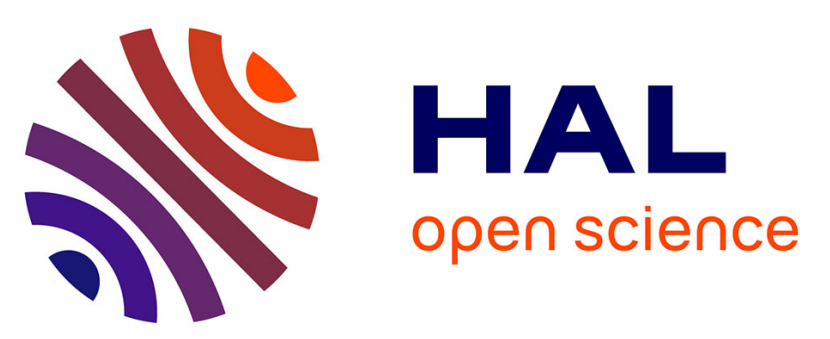

\title{
PROPAGATION D'EXCITATIONS ACOUSTIQUES NON LINÉAIRES DANS DES MATÉRIAUX DOTÉS DE MICROSTRUCTURES
}

\author{
M. Sayadi, Jean Pouget
}

\section{- To cite this version:}

M. Sayadi, Jean Pouget. PROPAGATION D'EXCITATIONS ACOUSTIQUES NON LINÉAIRES DANS DES MATÉRIAUX DOTÉS DE MICROSTRUCTURES. Journal de Physique Colloques, 1990, 51 (C3), pp.C3-219-C3-230. 10.1051/jphyscol:1990323 . jpa-00230751

HAL Id: jpa-00230751

https://hal.science/jpa-00230751

Submitted on 1 Jan 1990

HAL is a multi-disciplinary open access archive for the deposit and dissemination of scientific research documents, whether they are published or not. The documents may come from teaching and research institutions in France or abroad, or from public or private research centers.
L'archive ouverte pluridisciplinaire $\mathbf{H A L}$, est destinée au dépôt et à la diffusion de documents scientifiques de niveau recherche, publiés ou non, émanant des établissements d'enseignement et de recherche français ou étrangers, des laboratoires publics ou privés. 
PROPAGATION D'EXCITATIONS ACOUSTIQUES NON LINÉAIRES DANS DES MATÉRIAUX DOTÉS DE MICROSTRUCTURES

\author{
M.K. SAYADI et J. POUGET
}

Laboratoire de Mođélisation en Mécanique, associé au CNRS. Université Pierre et Marie Curie, Tour 66, 4, place Jussieu, F-75252 Resume Paris Cedex 05, France

Le probleme d'ondes non linéaires coupleses rotation-deplacement est présenté dans le cadre d'un modele de reseau cristallin presentant des dezres internes en rotation Cpar exemple cristaux avec groupes moléculairess. Sur la base du modele microscopique et de son approximation aux milieux continus, nous montrons l'existence d'ondes acoustiques non lineaires engendreses, via le couplage, par des ondes solitaires en rotation. L'important probleme d'un champ sinusoidal applique est etudie et montre une adaptation du systeme au champ exterieur. Les resultats sont illustrés par des simulations numeriques.

\title{
Abstract
}

The noninear wave propagation in crystal involving coupled motions in displacement and rotation is presented. The crystal is modelled by a one-dimensional monoatomic chain equipped with rotatory microstructures (for instance, crystal with molecular groups). On the basis of the microscopic model and its continuum approximation, the propagation of nonlinear acoustic waves generated by salitary waves in rotation is examined for two simple configurations. The influence of an applied sinusoidal field upon the nonlinear waves is examined and a transient motion of the rotation-deformation is placed in evidence. The relevant results are illustrated by means of numerical simulations.

\section{INTRODUCTION}

Nous nous proposons dans ce travail d'examiner le probleme de la propagation d'excitations acoustiques non linéaires dans des cristaux dotés de microstructures. En effet, certains materiaux presentent, outre leur deformation usuelle, des degres de liberte internes que seule une description cinematique suffisamment fine permet de mettre en evidence. G'est le cas des cristaux moleculaires pour lesquels les particules en interaction au sein du milieu ne peuvent pas etre reduites a un point dote d'une masse; mais en chaque point un sroupe moleculaire effectue des mouvements de rotation de corps rigide de sorte qu'un moment cinetique peut etre défini. Les mouvements de miaro-rotation et de deformation du cristal sont bien evidemment couples et il en découle donc la propagation d'ondes couplés en rotation-deformation.

Toutefois, le cristal est assujetti a des interactions interatomiques qui dependent de la miaro-physique du systeme, mais permettent des mouvements de rotation de larges amplitudes des groupes moleculaires (mouvement de renversement des molécules. De tels comportements se rencontrent dans les cristaux liquides [ 1], les longues chaines de macromolecules 〈par exemple 1'ADN) [2, 31 ou les cristaux moleculaires ferroelectriques [4, 5]. Pour ces derniers, le nitrite de sodium en est un bon exemple et dans co cas le mouvement de potation des groupes moleculaires decrit, la migration d'une paroi separant deux domaines ferroélectriques à $180^{\circ}$ [ 2 ].

Il decoule dong que le comportement non lineaire lie aux mouvements de micro-rotation engendre, par couplage, des ondes acoustiques non lineaires dont les caracteristiques, vitesses par exemple, sont fonctions de l'excitation non linéaire en rotation. Dans ce but, nous construisons un modele de réseau cristallin unidimensionnel incluant des interactions dipole-dipole 
entre sroupes moleculaires. Une telle approche est certainement tres approprié pour modeliser les mouvements relatifs aux degres de liberte internes et leurs couplases avec la deformation du cristal. Cependant le systeme d'equations ainsi obtenu est difficilement exploitable, aussi devons-nous requerir a l'approximation des milleux continus. Ceci condult a un systeme d'equations aux derivees partielles couplees dont nous pouvons trouver certaines classes de solutions du type onde localisee en rotation-deformation que nous decrivons par des solitons $t$ 6l. Les differents resultats sont fllustres par des simulations numeriques et le probleme de l'influence d'un champ appliqué est esalement tratte.

Le travail est organise de la maniere suivante : le paragraphe 2 est consacré a la construction d'un modele de reseau doté de microstructures. Le cas d'une configuration est examine au paragraphe 3 ; nous donnons les equations du systeme, des solutions d'ondes solitaires en rotation-deformation sont examinees analytiquement et numeriquement. Une seconde configuration est etudiee au paragraphe 4 montrant egalement des ondes solitaires en rotationdeformation. Le parasraphe 5 concerne plus particulierement le probleme de linfluence d'un champ exterieur sinusoldal sur le mouvement des ondes non linéaires. Finalement des conclusions et extensions du modele sont donnés au paragraphe 6 .

\section{DESCRIPTION DU MODELE}

Nous considerons une chaine monoatomique unidimensionnelle, constituee de $N$ cellules cristallines dotees chacune d'un moment d'inertie et d'un dipole electrique microscopique de module constant, indiquant, lorientation de chaque sraupe moleculaire. A titre d'exemple, nous pouvors citer le cristal ferroelectrique nitrite de sodium NaNO2 [ 71. Nous supposerons que $N$ est suffisamment grand afin de negliger les effets de bord et que ces cellules restent dans le plan $x O y$, ce que montre la figure 1; nous distinguerons alors trois types de mouvements au sein de chaque noeud $n$ de la chaine atomique : ( $i$ ) les deplacements longitudinaux du centre de masse de la cellule cristaline. notés $u_{n}$ (ii) les deplacements transwersaux de ce meme point, notes $v_{n}$ et (iii) les mouvements de rotation de corps rigide du groupe moleculaire ou du dipole electrique, reperre par les angles $\theta_{n}$ et $\varphi_{n}$.

Ces mouvements resultent des forces interatomiques agissant sur le réseau cristallin, solent : (i) les interactions atomiques entre les premiers voisins (interactions de courte et longue portés). (ii) les interactions mutuelles entre les dipoles electriques microscopiques, du type electrostatique et (iii) L'interaction electrostatique due au champ externe E.

Si de plus nous supposons que les deplacements demeurent infinitesimaux, alors que les rotations peuvent avoir de grandes amplitudes, nous ecrirons l'Hamiltonien du systeme comme sult [ 7]:

ou nous avons pose :

$$
H=K+W_{\text {Lat }}+W_{\text {Dip }}+W_{\text {Int }}+W_{E \times t^{\prime}}
$$

$$
\begin{gathered}
K=\frac{1}{2} \sum\left[M\left(\dot{u}_{n}^{2}+\dot{v}_{n}^{2}\right)+I \dot{e}_{n}^{2}+J \dot{\varphi}_{n}^{2}\right], \\
w_{L a t}=\frac{1}{2} \sum\left[K_{L}\left(u_{n+1}-u_{n}\right)^{2}+K_{T}\left(v_{n+1}-v_{n}\right)^{2}\right], \\
w_{D i p}=\sum\left[E\left(p_{n} \cdot p_{n+1}-3 p_{n}^{x} p_{n+1}^{x}\right)+n p_{n}^{x} p_{n+1}^{x}+v p_{n}^{y} p_{n+1}^{y}\right], \\
w_{I n t}=\frac{-3 E}{a} \sum\left[\left(p_{n} \cdot p_{n+1}-3 p_{n}^{x} p_{n+1}^{x}\right)\left(u_{n+1}-u_{n}\right)+\left(p_{n}^{x} p_{n+1}^{x}+p_{n}^{y} p_{n+1}^{y}\right)\left(v_{n+1}-v_{n}\right)\right], \\
w_{E x t}=P_{o L} \sum p_{n} \cdot E .
\end{gathered}
$$

L'expression (2.a) represente l'energie cinetique, ou $M$ est la masse de cellule cristalline, I et $J$ les moments d'irertie du groupe moleculaire, 
correspondant respectivement aux rotations $\theta_{n}$ et $\varphi_{n}$. L'enersie de deformation elastique est donnee par (2.b) dans laquelle $K_{L}$ et $K_{T}$ sont les constantes de rigidite longitudinale et transversale. L'expression (2.c) donne l'interaction entre les dipoles a laquelle s'ajoutent des termes d'arisotrople sutvant les axes $O x$ et $O y$, ces termes etant caracterises respectivement par les coefficients $\nu$ et $n$. Nous notons :

$$
\underline{P}_{n}=P_{0} P_{n}, \quad p_{n}^{x}=p_{n} \cdot \underline{x}, \quad p_{n}^{y}=p_{n} \cdot y \text { et } \quad P_{0}^{2}=4 \pi \varepsilon \varepsilon_{0} \alpha^{3} \text {. }
$$

ou a est le parametre du resseau et

$$
\mathrm{E}_{n}^{\mathrm{T}}=\left(\cos \theta_{n} \cos \varphi_{n}, \sin \theta_{n} \cos \varphi_{n}, \sin \varphi_{n}\right),
$$

L'equation (2.d) definit les interactions entre dipsles et deplacements, cette interaction est du type electrostrictif. Enfin, l'energie due au champ electrique exterieur est exprimé par (2.e).
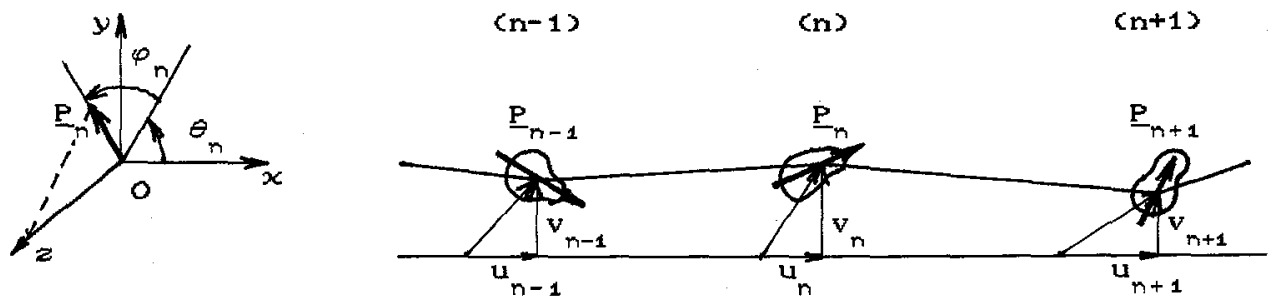

Fig. 1 - Chaine monoatomique equipee de dipoles electriques microscopiques

A ce stade, nous allons examiner deux conflgurations particulieres; ce sont les cas où $\varphi_{n}=0$, configuration A cles dipoles tournent autour de l'axe $0 z)$, et où $\theta_{n}=\pi / 2$, configuration $B$ (la rotation des dipoles se fatt autour de l'axe $O x$.

\section{ETUDE DE LA CONFIGURATION A}

\subsection{Equations discretes du mouvement}

Les equations adimensionnalisees du mouvement de la chaine monoatomique sont obtenues a partir de la relation (1) de l'Hamiltonien et s'earivent :

$$
\left\{\begin{array}{l}
u_{n}-c_{L}^{2}\left(u_{n+1}-2 u_{n}+u_{n-1}\right)=\frac{1}{2} \alpha\left[\cos \theta_{n+1}-\cos \theta_{n-1}\right], \\
v_{n}-c^{2}\left(v_{n+1}-2 v_{n}+v_{n-1}\right)=-\frac{1}{2} \beta\left[\sin \theta_{n+1}-\sin \theta_{n-1}\right], \\
\Theta_{n}-2\left[\sin \left[\frac{\theta_{n+1}-\theta_{n}}{2}\right]-\sin \left[\frac{\theta_{n}-\theta_{n-1}}{2}\right]\right]=\frac{1}{2} \chi\left[\sin \left(\frac{\theta_{n+1}+\theta_{n}}{2}\right]+\sin \left[\frac{\theta_{n}+\theta_{n-1}}{2}\right]\right] \\
+\frac{1}{2} \alpha\left(u_{n+1}-u_{n-1}\right) \sin \theta_{n}+\frac{1}{2} \beta\left(v_{n+1}-v_{n-1}\right) \cos \theta_{n}-\operatorname{Fos}\left(\theta_{n} / 2\right),
\end{array}\right.
$$

où nous avons posé :

$$
\left\langle U_{n}, v_{n}\right)=2\left(u_{n}, v_{n}\right) \sqrt{\frac{M}{I}}, \quad \theta_{n}=2 \theta_{n}, \quad \tau=t \sqrt{\frac{\varepsilon-n-V}{2 I}},
$$


et où :

$$
\left\{\begin{aligned}
x & =-4 \frac{3 \varepsilon-n+\nu}{\varepsilon-n-\nu}, & \alpha & =\frac{3}{2} \beta=\frac{18 \varepsilon}{a(\varepsilon-n-\nu)} \sqrt{\frac{T}{M}}, \\
F & =4 \frac{\mathrm{P}}{\varepsilon-n-\nu}, & \left(C_{L}^{2}, C_{T}^{2}\right) & =\frac{2 I}{M(\varepsilon-n-\nu)}\left(K_{L}, K_{T}\right) .
\end{aligned}\right.
$$

Ici nous avons suppose que $\varepsilon-n-\nu>0$ et en plus que $\theta_{n+1}-\theta_{n}=0(0)$.

Il est clair que ces equations sont non lineaires; de plus, en raison de la presence des termes de couplages, leur resolution analytique s'avere tres complexe, voire impossible. Néanmolns, elle peut se faire numeriquement en considerant des conditions initiales et aux limites appropriees. Le modele continu s'impose alors pour mieux comprendre la dynamique d'un tel systeme.

\subsection{Equations du modele continu}

Supposons maintenant que le parametre du reseau a est tres faible devant la longueur caractéristique des processus dyramiques clongueur d'onde ou epaisseur de la parol de domaine; ceal nous permet de developper toutes les quantites discretes en termes de series de Taylor autour de la position $\mathrm{X}=\mathrm{n}=\mathrm{x} / \mathrm{a}$. Nous dedulsons au premier ordre, en l'absence du champ electrique exterieur et dans l'hypothese $\chi \ll 1$, le système d'equations aux derivées partielles sulvant :

$$
\left\{\begin{array}{l}
U_{\tau \tau}-c_{L}^{2} U_{x x}=\alpha(\cos \theta)_{x}, \\
v_{\tau \tau}-c_{T}^{2} v_{x x}=-\beta(\sin \theta)_{x}, \\
\theta_{\tau \tau}-\theta_{x x}=x \sin \theta+\alpha U_{x} \sin \theta+\beta V_{x} \cos \theta .
\end{array}\right.
$$

La structure de ces equations pressente un grand interet, car les deux premieres sont celles de propagation d'ondes acoustiques linealres, modifiees par la présence des dipoles et la derriere est une equation non lineaire du type sine-Gordon couplee avec les deplacements. Le présent modele peut stre compare a celui d'un milieu continu oriente et non linealre $I$ 81, ausisi bien qu'à celui des cristaux micropolaires elastiques [ 9 ].

Nous nous proposons maintenant de chercher des solutions sous forme d'ondes propagatives, pour lesquelles $U, v$ et $\theta$ sont fonctions de la seule variable de phase $\xi=\mathbf{x}-\mathbf{x}_{0}-C_{T}$, ou $\mathrm{G}$ est la vitesse de phase et $x_{0}$ la position inftiale de londe. Ainsi les deformations $U_{\xi}$ et $v_{\xi}$ peuvent etre obtenues en fonction de l'angle $\theta$ :

$$
\mathrm{U}_{\xi}=-\frac{\alpha \cos \theta}{\mathrm{c}_{L}^{2}-\mathrm{c}^{2}}+\mathrm{A}, \quad \mathrm{V}_{\xi}=\frac{\beta \sin \theta}{\mathrm{c}_{T}^{2}-\mathrm{c}^{2}} \text {. }
$$

où l'on a suppose que $C^{2}$ differe de $C_{L}^{2}$ et $G_{T}^{2}$ et ou $A$ est une constante d'integration arbitraire. Si nous reportons maintenant les expressions (7) dans l'equation gouvernant le mouvement de $\Theta$, il vient que" :

$$
\begin{gathered}
\left(\mathrm{c}^{2}-1\right) \Theta_{\xi \xi}=\tilde{x}\left(\sin \Theta-\frac{\delta}{2} \sin 2 \Theta\right), \\
\ddot{x}=x+\alpha A, \quad \tilde{x} \delta(C)=\frac{\alpha^{2}}{\mathrm{c}_{L}^{2}-\mathrm{c}^{2}}-\frac{\beta^{2}}{\mathrm{c}_{T}^{2}-\mathrm{G}^{2}}
\end{gathered}
$$

Nous constatons alors que le probleme d'ondes solitaires du systeme ( 6 ) se ramene simplement a la resolution de l'equation (8.a), qui est une equation differentielle ordinalre, non lineaire. L'existence de ces ondes localisees depend des parametres $\tilde{x}$, $\delta$ et $C$; nous nous limitons 1 c1 au cas ou $\tilde{x}>0$, $\delta \leq 1$ et $|\mathrm{C}|<1$, et nous ecrivons la solution comme sult : 
où

$$
\varepsilon=\lambda \varepsilon_{0}, \quad \lambda^{2}=\frac{1}{1-\delta}, \quad z_{0}^{2}=\frac{1-\mathrm{c}^{2}}{\tilde{x}} \text {. }
$$

Notons ici que $\varepsilon_{\mathrm{o}}$ est une longueur caractéristique proportionnelle a l'epaisseur de la paroi de domaine et que la solution (9.a) satisfait les conditions de stabilite [10].

L'etat mecanique du cristal est alors donne par les deformations en elongation et en cisalllement qui s'écrivent :

$$
\mathrm{u}_{\xi}^{ \pm}=\mathrm{u}_{\alpha \xi}^{ \pm}+\frac{2 a}{\mathrm{c}_{L}^{2}-\mathrm{c}^{2}}\left[1-\frac{1}{\lambda^{2} \operatorname{sh}^{2}(\xi / \theta)+1}\right], \quad \mathrm{v}_{\xi}^{ \pm}= \pm \frac{2 \beta \lambda}{\mathrm{a}_{\mathrm{T}}^{2}-\mathrm{G}^{2}} \frac{\operatorname{sh}(\xi / \ell)}{\lambda^{2} \operatorname{sh}^{2}(\xi / \phi)+1} .
$$

où la constante d'integration A est remplacee par la deformation $U_{o \xi}=U_{\xi}(0)$. Nous remarquons que la deformation $U_{\xi}$ demeure constante quand $\xi \longrightarrow \pm \infty$.

Il convient d'ajouter qu'ayant un milieu mecaniquement linéaire, il est possible d'engendrer, par couplase electromécanique, une onde solltaire de contrainte et de déformation par le biafs du mouvement de paroi entre deux domaines ferroelectriques. L'epaisseur de la paroi s'etend sur une dizaine de particules, ce qui correspond a quelques centiemes de $\mu \mathrm{m}$ dans le cas du nitrite de sodium observé à l'alde d'un microscope electronique [11].

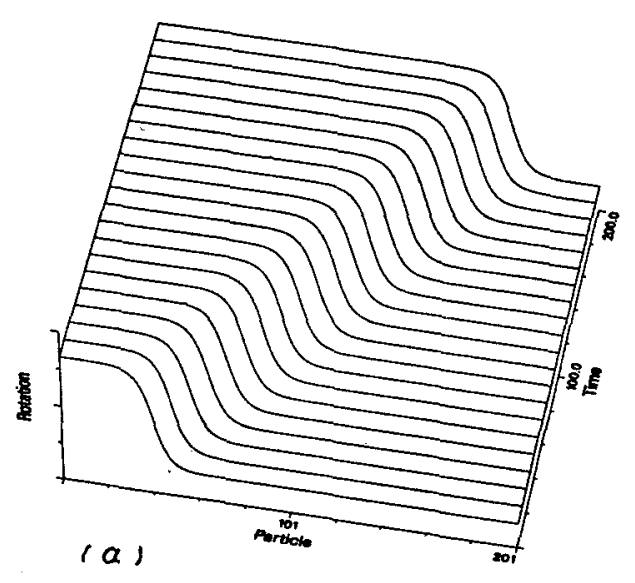

Fig. 2 - Configuration A, Cas soliton en rotation, (b) soliton en elongation et (c) soliton en cisaillement.
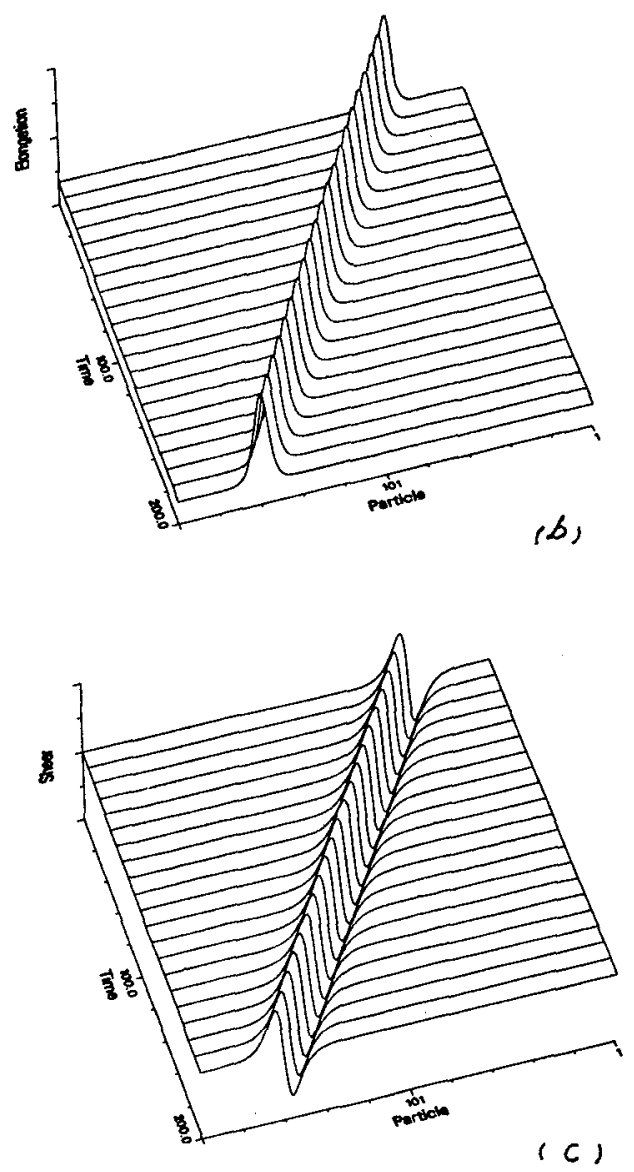


\subsection{Simulations numeriques}

Le schema numerique utilise est directement donne par les equations discretes (4) pour lesquelles les solutions definies precedemment sont considérees comme conditions inttiales. La floure 2.a donne le mouvemert de rotation des dipoles (mouvement d'une paroi de domaine a 180') : c'est une onde solitalre en forme de "front", qui represente la transition de l'etat $\theta^{-}=\pi$ quand $x \rightarrow-\infty$, a l'etat $\theta^{-}=-\pi$ quand $\mathrm{X} \rightarrow+\infty$. Le couplage non lineaire permet ainsi d'engendrer des ondes acoustiques en compression-extension et en cisallement accompagnant le mouvement de renversement des dipoles electriques, respectivement representes en figures $2 . b$ et $2 . c$.

\section{ETUDE DE LA CONFIgURATION B}

\subsection{Equations discretes du mouvement}

En falsant $\theta=\pi / 2$ dans l'expression (1) de l'Hamiltonien, et en considerant le changement de variable suivant :

(11.a)

$$
U_{n}=2 u_{n} \sqrt{\frac{M}{J}}, \quad \tau=t \sqrt{-\frac{2 \varepsilon+\nu}{2 J}}, \text { avec } \quad 2 \varepsilon+\nu<0
$$

où nous definissons :

$$
\begin{cases}\mu=-\frac{\nu}{2 \varepsilon+\nu}, & z=\frac{\sigma \varepsilon}{\mathrm{a}(2 \varepsilon+\nu)} \sqrt{\frac{J}{M},} \\ f=-\frac{2 \mathrm{P}}{\mathrm{O}} \mathrm{E} & \hat{\mathrm{G}}_{\mathrm{Z}}^{2}=\frac{-2 \mathrm{~J}}{\mathrm{M}(2 \varepsilon+\nu)} \mathrm{K}_{L} .\end{cases}
$$

nous ecrivons les equations du mouvement sous la forme sulvarte :

$$
\left\{\begin{aligned}
\dot{U}_{n}- & \hat{C}_{L}^{2}\left(U_{n+1}-2 U_{n}+U_{n-1}\right)=-2 \sin \left[\frac{\varphi_{n+1}-\varphi_{n-1}}{2}\right] \sin \left[\frac{\varphi_{n+1}-2 \varphi_{n}+\varphi_{n-1}}{2}\right], \\
\ddot{\varphi}_{n}- & {\left[\sin \left(\varphi_{n+1}-\varphi_{n}\right)-\sin \left(\varphi_{n}-\varphi_{n-1}\right)\right]=\mu\left[\sin \left(\varphi_{n+1}+\varphi_{n}\right)+\sin \left(\varphi_{n}+\varphi_{n-1}\right)\right] } \\
& -x\left[\left(U_{n+1}-U_{n}\right) \sin \left(\varphi_{n+1}-\varphi_{n}\right)-\left(U_{n}-U_{n-1}\right) \sin \left(\varphi_{n}-\varphi_{n-1}\right)\right]+f \cos \varphi_{n} .
\end{aligned}\right.
$$

L'equation souvernant les deplacements transversaux n'est pas prise en compte pulsqu'elle ne joue aucur role dans cette situation. Remarquons ausisi que les termes de couplage présents dans le système (12) different de ceux du systeme (4) de la configuration A.

\subsection{Equations du modele continu}

En procedant d'une maniere analogue à la premiére conflguration, l'approximation continue du systeme d'equations aux differences rinies est obtenue pour des processus dynamiques lentement variables en espace. Les equations alnsi déduites s'écrivent :

$$
\left\{\begin{array}{l}
\tilde{\mathrm{U}}_{\tau \tau}-\hat{\mathrm{C}}_{\mathrm{L}}^{2} \tilde{\mathrm{U}}_{\mathrm{xx}}=-\frac{1}{2} \alpha\left(\left(\phi_{\mathrm{x}}\right)^{2}\right)_{\mathrm{x}}, \\
\phi_{\tau \tau}-\phi_{\mathrm{xx}}=x \sin \phi-\alpha\left(\tilde{U}_{\mathrm{x}} \phi_{\mathrm{x}}\right)_{\mathrm{x}^{\prime}}
\end{array}\right.
$$

où :

$$
\tilde{\mathrm{U}}=2 \mathrm{U}, \quad \phi=2 \varphi, \quad \alpha=\frac{\not \ell}{2} \text { et } \quad x=4 \mu \ll 1 .
$$

En negligeant le couplage, nous retrouvons l'equation d'ondes acoustiques pour le deplacement longitudinal, et celle de sine-Gordon pour la rotation; a cause de ce couplage, la structure de ces equations devient tres complexe et leur résolution fort dellcate. 
Les solutions d'ondes solitaires peuvent etre egalement obtenues sous forme d'ondes propagatives, fonctions de la variable de phase $\xi$. Nous pouvons alors substituer la deformation $\widetilde{U}_{\xi}$ dans l'equation regissant le mouvement de $\phi$; et nous obtenons :

(15.a)

$\tilde{U}_{\xi}=\frac{1}{2} \frac{\alpha}{\hat{\mathrm{C}}_{L}^{2}-\mathrm{C}^{2}}\left(\phi_{\xi}\right)^{2}+A$,

et par sulte :

(15.b)

$$
\frac{3}{2} \frac{\alpha^{2}}{\hat{C}_{L}^{2}-c^{2}}\left[\phi_{\xi}\right]^{2} \phi_{\xi \xi}-\left(1-c^{2}-\alpha A\right) \phi_{\xi \xi}-\chi \sin \phi=0 .
$$

Nous supposons que le coefflaient de couplage a est non nul; dans ces conditions, l'equation (15.b) admet une integrale premiere qui s'ecrit :

(16.a)

où nous avons pose :

$$
\left(\phi_{\zeta}\right)^{4}-2 p a\left(\phi_{\zeta}\right)^{2}+b+p \cos \phi=0 \text {, }
$$

(16.b)

$$
\left\{\begin{array}{l}
\zeta=\xi \sqrt[4]{\left|\frac{x}{\gamma}\right|,} \quad a=\frac{1-c^{2}-\alpha A}{4 x} \sqrt{\left|\frac{x}{\gamma}\right|,} \\
p=\operatorname{sign}\left[\frac{x}{\gamma}\right], \quad \text { avea } \quad \gamma=\frac{3}{8} \frac{a^{2}}{\hat{c}_{L}^{2}-c^{2}},
\end{array}\right.
$$

Dans ce qui précede, $c^{2}$ differe de $\hat{C}_{L}^{2}$, A et b sont des constantes d'intégration arbitraires.

Dans le cas le plus seneral, les solutions de l'equation (16.a) s'expriment a l'aide des intégrales elliptiques de premiere et troisieme espece. Nous allons donc examiner un cas simple conduisant a une onde solitalre pour laquelle nous avons $a=\sqrt{2}$ et $b=p=1$, solt :

par consequent :

$$
\left[\phi_{\zeta}\right]^{2}=\sqrt{2}[1-|\sin (\phi / 2)|]
$$

(18.a)

$$
\varphi^{ \pm}= \pm 4 \operatorname{sign}(\xi)\left[\operatorname{Arc} \operatorname{tg}[\exp (|\xi| / z+\Delta)]-3 \frac{\pi}{8}\right],
$$

et.

(18.b)

(18.c)

$$
\begin{gathered}
\mathbf{u}_{\xi}^{ \pm}=\frac{1-\mathrm{c}^{2}}{x}+\frac{32 \gamma}{3 x z^{2}}\left[2 \operatorname{sech}^{2}[|\xi| / \ell+\Delta]-3\right] \\
i=2 \sqrt[4]{\frac{2 \gamma}{x}}=\sqrt{\frac{1-\mathrm{c}^{2}-\alpha A}{x}} \text { et } \quad \Delta=\operatorname{Arg} \operatorname{sh} 1 .
\end{gathered}
$$

Nous remarquons qu'une telle solution existe si $|\mathrm{C}|<$ inf(1 $\left.-\alpha A, \hat{\mathrm{C}}_{\mathrm{L}}\right)$, $\chi>0$ et $\alpha \neq 0$, ce qui conduit, en l'absence des termes de couplages, a l'equation usuelle de sine-Gordon. La constante d'Integration A, figurant dans l'expression (18.b) de la deformation, a ete eliminé en utilisant la relation (18.c); dans cette derniere $i$ est une longueur caracteristique lié a l'épalsseur de la parai par $e=\pi \ell$.

La solution (18.a) esst telle que $p^{ \pm}$transite de $\mp \pi / 2$ quand $\xi \longrightarrow-\infty$, a $\pm \pi / 2$ quand $\zeta \longrightarrow+\infty$; nous retrouvons donc une situation assez similaire a celle d'une chaine compressible de dipoles [12], ou bien a celle d'un modele d'Heisenberg pour les chaines de spins [13].

\subsection{Resultats numeriques}

Afin de verifler la stablite de ces ondes locallsées aussi bien que l'approximation continue, nous avons considere une simulation numerique au 
moyen des equations discretes (12). les resultats sont représentes sur la figure 3.a pour la rotation et sur la figure 3.b pour la deformation; cette derniere est accompasné de radiations de phonon et d'une perte d'amplltude, leur influence sur la rotation est moindre. En fait, ces effets sont dus a l'approximation continue qui induit un manque d'information par rapport au modele discret. Cette simulation est la melleure pour des vitesses de phase avolsinant l'unite ce qui est confirme par l'expression (18.b) de la deformation; en effet, cela respecte les hypotheses de faibles variations spatiales. Aussi cette vitesse peut etre inferieure ou superieure a 1.

\section{INFLUENCE D'UN CHAMP EXTERIEUR SUR LE MOUVEMENT DES EXCITATIONS NON LINEAIRES}

\subsection{EQUATIONS}

Le probleme que nous nous proposons d'examiner maintenant est le mouvement transitoire de l'onde localisee, initialement au repos, quand un champ electrique non nul est soudainement applique a l'1nstant $\tau=\tau$. Nous determinerons alors les etats électriques et mécaniques de la paroi sous l'influence du ahamp. Physiquement, la présence de ce champ favorise la croissance d'un domaine au detriment de l'autre, ce qui est un moyer de ramener une structure a deux domaines a une autre n'en comportant qu'un seul. En supposant que le mouvement de la parol a lieu dars le plan $x O y$, seule 1"equation en $\theta$ est modtfite et s'earit:

$$
\theta_{\tau \tau}-\theta_{\mathbf{x} \mathbf{x}}+\gamma \theta_{\tau}=x \sin \theta+\alpha U_{\mathbf{x}} \sin \theta+\beta V_{\mathbf{x}} \cos \theta-F(\tau) \cos (\theta / 2)
$$

Nous avons introduit dans cette equation un processus dissipatif et un champ applique dependant de la rotation elle-meme. En revanche, nous n'avons pas inclus de processus de dissipation lie a la deformation elastique de la chaine, bien que la viscosite soit le modele le plus appropriee, celle-ci est difficile a introduire correctement sur le plan microscopique.

Les termes supplementaires de l'equation (18) sont consideres comme de petites perturbations agissant sur le systéme couple et ont pour effet de moduler la position de londe solltalre (translation), d'alterer son epaisseur et de changer sa forme par la presence des radiations de phonon. Ainsi la parol initialement au repos depend maintenant du temps. Desormais et par souci de simplification, ces radiations seront ignorees.
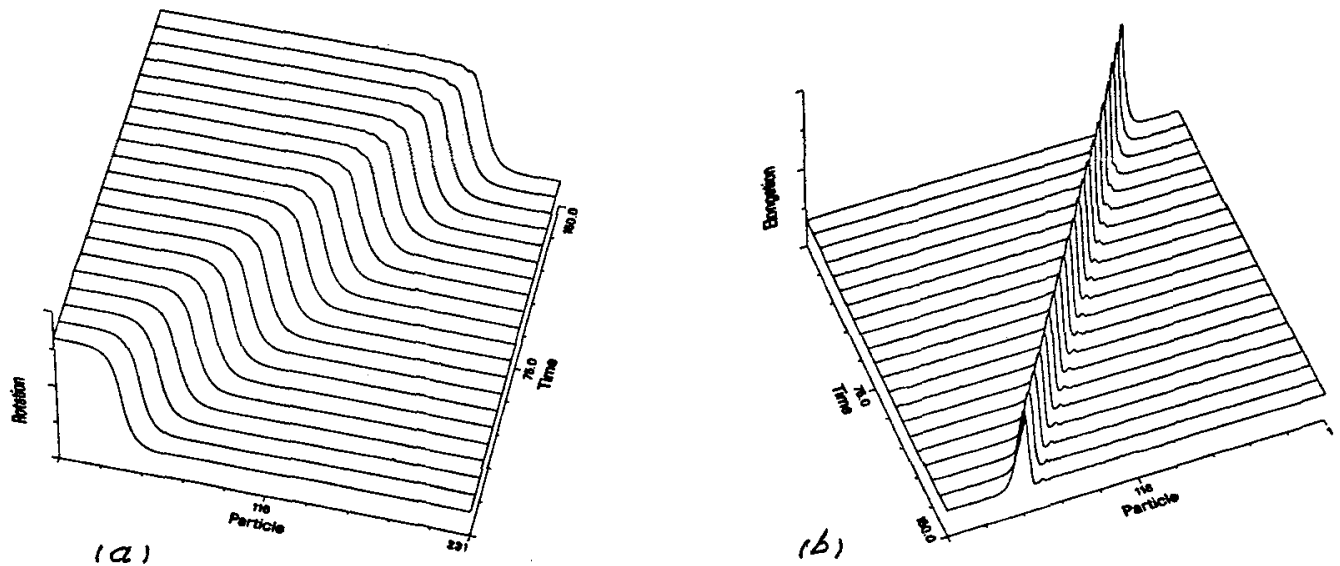

Fis. 3 - Configuration B. (a) soliton en rotation et (b) soliton en élonsation. 
Un schema de perturbation a ete developpe en consequence; 11 est bass sur l'hypothese de la variation adiabatique du temps 17, 141, qui consiste à remplacer la variable de phase precédente par $\xi=\mathrm{X}-\omega(\tau)$, où $2 \omega(\tau)$ est. la position de l'onde perturbee et $C(\tau)=\omega(\tau)$ sa celerite.

Grace a une consideration energetique simple, 11 est possible de definir ces grandeurs en fonction du temps; en effet, la methode repose sur le falt que les solutions (9.a) et (10) sont fonctions des vartables $(x, w(\tau)$, $\tau)$ et sont contraintes a satisfaire un principe variationnel associe au Lagrangien du systeme. Ce princlpe est verifie pour tout mouvement virtuel de deplacement et de rotation, soient $\delta U, \delta V$ et $\delta \Theta$. Ces mouvements sont dus a la position virtuelle de londe, qui elle-meme est provoquee par les travaux virtuels du champ exterieur et de la dissipation. La position w peut etre alors traité comme une nouvelle variable dynamique; ce qui conduit a l'equation du mouvement suivante:

$$
\frac{d}{d \tau}\left[\frac{\partial E}{\partial C}\right]-\frac{\partial L}{\partial w}=Q_{F}+Q_{D}
$$

ou $\mathscr{L}$ est le Lagrangien defini a partir de la solution non perturbee (U, $v$, $\Theta$ ), $Q_{F}$ la force seneralisee des efforts exterieurs et $Q_{D}$ celle de la dissipation, ces grandeurs s'ecrivent comme sult :

$$
\begin{gathered}
\mathscr{E}(\tau)=-8 \sqrt{\hat{x}\left(1-\mathrm{C}^{2}(\tau)\right)}, \\
Q_{F}(\tau)= \pm 4 \mathrm{~F}(\tau),\left(\text { selon } \theta^{ \pm}\right), \\
Q_{D}(\tau)=-8 \gamma(1+\varepsilon) \mathrm{C}(\tau) \sqrt{\frac{\hat{x}}{1-c^{2}}} .
\end{gathered}
$$

Dans les equations (21) nous avons suppose que $\delta(G)=b(0) \ll 1$ et nous avons pose :

$$
\hat{x}=\tilde{x}\left[1-\frac{\delta}{3}-2 \varepsilon\right], \quad 2 \varepsilon \tilde{x}=\alpha A
$$
devient :

En utilisant les expressions (21.a-c), l'equation pour la position w( $\tau)$

$$
\begin{aligned}
& \frac{d}{d \tau}\left[\frac{c}{\sqrt{1-c^{2}}}\right]+\hat{\gamma} \frac{c}{\sqrt{1-G^{2}}}=\hat{F}(\tau), \\
& \hat{\gamma}=\gamma(1+\Leftrightarrow), \quad \hat{F}(\tau)= \pm \frac{F(\tau)}{2 \sqrt{x}}
\end{aligned}
$$

Nous avors bien evidemment une equation relativiste pouvant etre aisement résolue. En particulier, si $\gamma$ est nul et $F$ constant dans le temps, nous retrouvons lequation de mouvement d'une particule relativiste de masse unite au repos, uniformement acceléree par $F$ [15].

\subsection{Solutions numeriques}

La simulation numérique de ce probleme est lllustrée par la flgure 4 , qui donne les solutions des etats de deformations en elongation et en cisaillement crigures $4 . a$ et $4 . \mathrm{b}$ ) et celle en rotation (rigure 4.c). L'onde est initialement au repos $\left\langle\tau\left\langle\tau_{0}\right\rangle\right.$ et amorce un mouvement progressif quand $F$ est appliquee a $\tau_{o}$; elle acquiert donc une vitesse periodique dependant a la fols de $F$ et de $\gamma$. La figure 5 donne la position et la vitesse de l'onde localisee en fonction du temps, alrisi que la trajectoire dans le plan des phases. De plus, ajoutons que les radiations de phonon dues aux termes perturbateurs sont difficilement perceptibles; en revanche les effets de la discretisation sont apparents au niveau de la vitesse. Ces resultats confirment la validite de 
notre methode de perturbation aussi bien que l'approximation continue.

\section{CONCLUSION}

Nous avons construit dans ce present travall un modele de reseau anharmonique, incluant des degres de liberte supplementaires inherents a la rotation de dipoles. Le systeme microscopique ainsi obtenu est alors a la fols non Hnealre (rotation de srandes amplitudes) et aussi dispersif cheterogeneite en espace des dipoless, 11 engendre en consequence des excitations acoustiques electromecaniques du type ondes solitaires; celles-ci sont intimement liees au mouvement de la parol separant deux domaines ferroelectriques et sont obtenues au moyen d'une approximation continue. Neanmoins, cette derniere s'accompagne dans certains cas d'une perte d'information par rapport au modele discret correspondart, et condult a des perturbations; une analyse de la stabilite peut etre menée pour mieux cerner la dynamique du réseau. De telles ondes non linéaires, dans la mesure ou leur stabilité est assuré, peuvent etre appliquees dans des dispositifs de traitement du signal cpar exemple, produit de convolution, diffraction d'ondes, compression d'impulsions etc ... .

Par alleurs, le modele peut etre etendu au cas ou les deux configurations $A$ et $B$ sont presentes et nous pouvons, par exemple, examiner la stabilite d'ure configuration relativement a l'autre. D'autre part, Le caractere discret du systeme engendre des phenomenes non lineaires particulierement interessants a etudier et qui n'existent pas dans le cas continu, tels que l'hysteresis ou la transition vers le chaos, quand nous appliquons un ahamp électrique dépendant du temps. Finalement, nous pouvons envisager l'extension de ce modele a un systeme bi-dimensionnel qui condult a de nouvelles structures non lineaires et a de nouveaux problemes de stabilité.
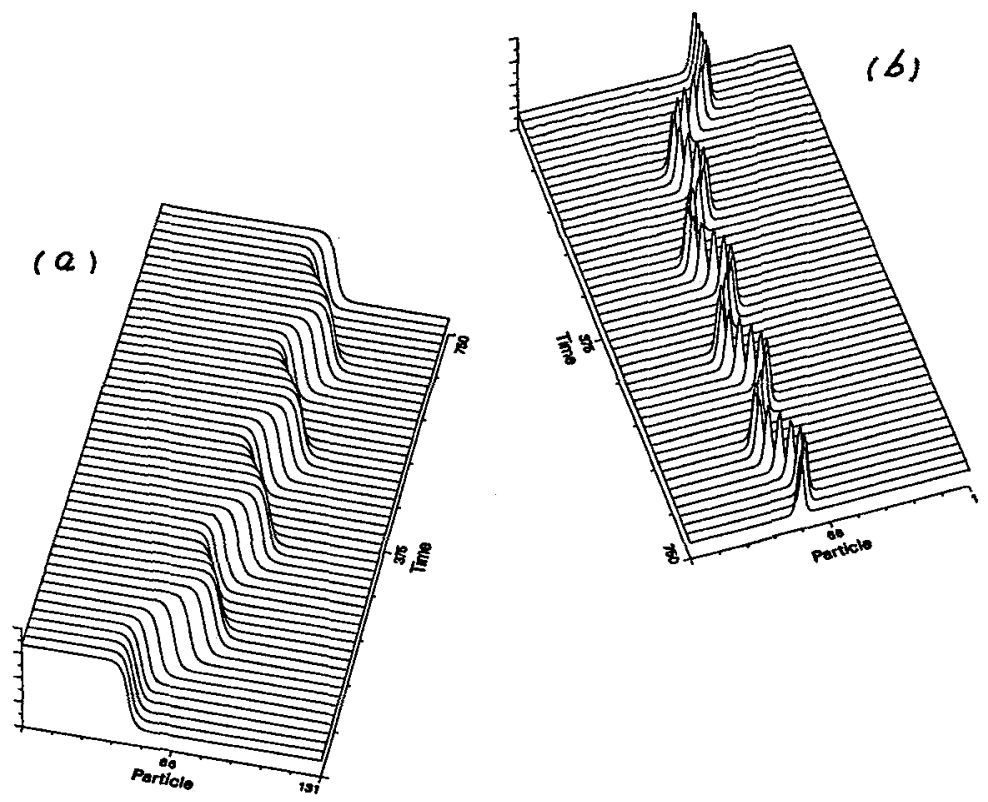

Fig. 4 - Influence d'un champ applique sur les ondes solitaires. (a) soliton en rotation, (b) soliton en flonsation et $(c)$ soliton en cisaillement.

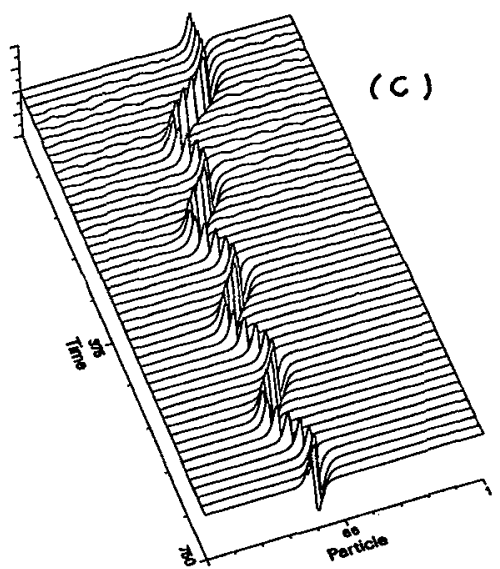




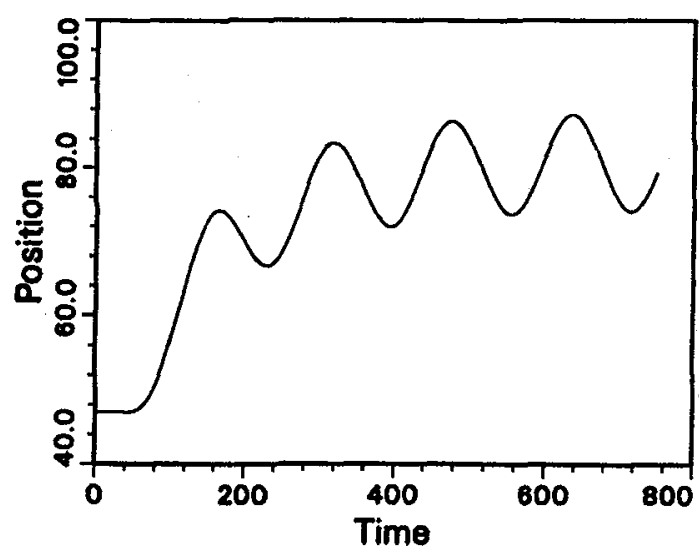

(a)

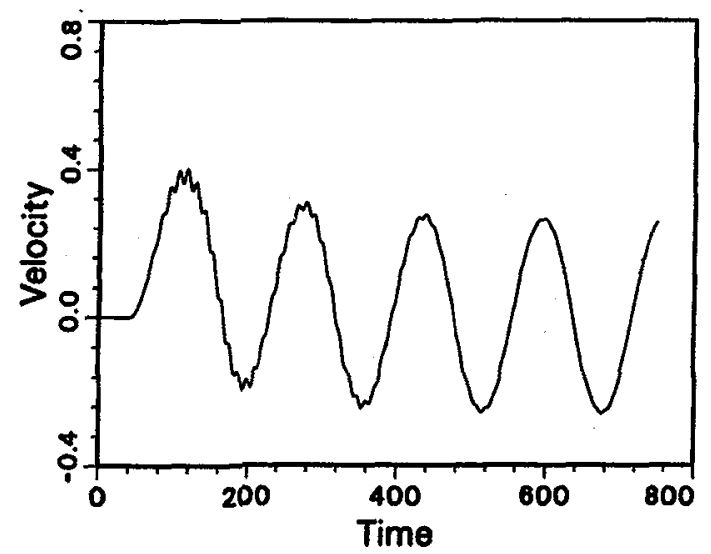

(b)

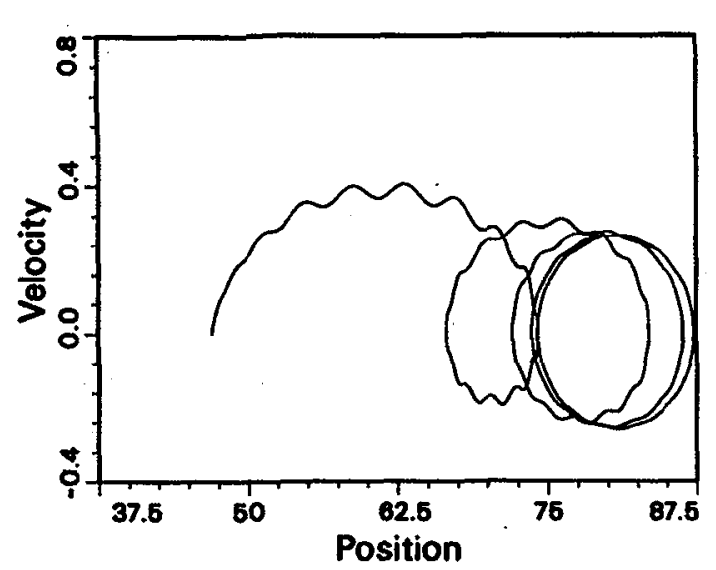

(c)

Fig. 5 - Parametres de ionde solitaire en fonction du temps, $(a)$ position de londe, (b) vitesse de l'onde et (c) trajectoire dans le plan des phases.

\section{REFERENCES}

11 E. MAGYARI - The inertia mode of the mechanicaliy generated solitons in nematic liquid crystals. Z. Phys. B-Condensed Matter 56, 1-3 (1984).

I 21 H.D. VEY-AHARON, T.J.SLUCKIN, P.L. TAYLOR \& A.J. HOPFINGER - Kink propagation as a model for poling in a poly cuinylidenefluoride). Phys. Rev. Bz1, 3700-3707 (1980).

I 31 S. YomosA - Soliton excitations in dioxyribonacleic acid (DNA) double helices. Phys. Rev. A27, 2120-2125 (1983).

I 4] W. KINASE, W. MAKINO \& K. TAKAHASHI - Theory of the domain wall in NaNOz and the relation with the incommensurate structure. Ferroelectrias 64 , 173-180 (1985).

I 5) A. ASKAR - A model for coupled rotation-dispiacement mode of certain molecular crystals, illustration for KNO3. J. Phys. Solids 34, 1901-1907 (1973).

I 61 A.C. NEWELL - The history of the soliton. Transaction of the ASME, J. of Appl. Mech. 50, 1127-1138 (1983).

I 7] J. POUGET - Nonlinear dynamics of lattice models for elastic media. in NATO-ASI serles, Vol. 225 "Physical Properties and Thermadynamic Behaviaur of Minerais", ed. by E.K.H. Salje, p 359 (D. Reidel Dordrecht, 1988).

I1 J. POUGET \& G.A. MAUGIN - Nontinear dynamics of oriented elastic solids I and II. J. of Elasticity Z2, 135 and 157 (1989). 
[ 9] G.A. MAUGIN \& A. MILED - Solitary waves in micropolar elastic crystals. Int. J. Eng. Sc1. 24, 1477-1499 (1986).

[101 A.J. CALlEGAR \& E.L. REISS - Nonlinear stability problem for the sineGordon equation. J. Math. Phys. 14, 267-276 (1973).

[11] S. SUZUKI \& M. TAKAGI - Topographic study in ferroelectric NaNOz crystals I. Structure of $180^{\circ}$ domain wall. J. Phys. Soc. Jpn. 30, 188-202 (1971).

[12] M. REMOISSENET - Nonlinear excitations in a compressible chain af dipoles. J. Phys. C : Solid state Physics 14, L335-L338 (1981).

[13] F. FIVEZ - On the continuum limit of a classical compressible Heisenberg chain. J. Phys. G : Solid state Physics 15, L641-L643 (1982).

[14] J. POUGET - Transient motion of a solitary wave in elastic ferroelectric. In Lecture Notes in Physics 249 "Trends in Applications of Pure Mathematics to Mechanies', ed. by E. Kroner and K. Klrchgassner, 156-153 cSpringer-Verlag, Berlin, 1985).

[15] J. POUGET \& G.A. MAUGIN - Influence of an external field on the motion of a ferroelectric domain wall. Phys. Lett. 109 A, $389-392$ (1985). 